\title{
Percutaneous Management of Chronic Central Venous Occlusive Disease
}

\author{
Matthew G. Gipson, MD ${ }^{1} \quad$ Rajan K. Gupta, MD ${ }^{1} \quad$ Mitchell T. Smith, MD ${ }^{1}$ \\ ${ }^{1}$ Department of Radiology, University of Colorado, Aurora, Colorado \\ Address for correspondence Matthew G. Gipson, MD, Mail Stop L954, \\ Semin Intervent Radiol 2015;32:57-60 \\ Leprino, 12401 East 17th Avenue, Room 540, Aurora, CO 80045 \\ (e-mail: matthew.gipson@ucdenver.edu).
}

Stenosis or occlusion of the central veins and/or vena cava can cause painful and debilitating symptoms for affected patients. Superior vena cava syndrome (SVCS) can develop acutely or progressively from external compression or intrinsic obstruction. The clinical signs and symptoms result from venous hypertension and reduced blood flow in the superior vena cava (SVC) or central veins emptying into the SVC. Depending on the anatomic location of the narrowing, clinical symptoms, and presentation will vary. Clinical signs include cyanosis, plethora, distention of subcutaneous veins, and edema of the upper extremities, head and neck. Edema may compromise laryngeal or pharyngeal function, causing dyspnea, stridor, cough, hoarseness, and dysphagia. ${ }^{1}$ Although there may be symptom overlap with congestive heart failure, a physical examination will usually delineate between the two diagnoses.

The etiology of SVCS varies and can be broadly grouped into benign and malignant causes, with most strictures of the caval system and venous tributaries being malignant. ${ }^{2,3}$ Nonsmall-cell lung cancer (NSCLC) is the most common malignant cause of SVCS, followed by small-cell lung cancer (SCLC) and non-Hodgkin lymphoma. ${ }^{4}$ The most common causes of benign venous stenosis involve the presence of an indwelling intravascular device (i.e., catheters, pacemaker/defibrillators, ports). Arteriovenous fistula related obstruction, mediastinal fibrosis, and prior thoracic external bean radiation therapy are additional benign causes.

Treatment of central venous occlusions depends on the etiology and clinical presentation. The ultimate goal of therapy is to correct the obstruction and alleviate the symptoms. Conservative measures depend on the etiology and include limb elevation, steroids, anticoagulation, chemotherapy and/or radiation therapy, and diuretics. ${ }^{5}$ Interventional therapies include surgical bypass and/or endovascular stent placement. Guide wire recanalization and endovascular stenting is a minimally invasive procedure that can be performed on an outpatient basis with standard equipment available in an angiography suite. The procedure below describes the authors approach to treating chronic central venous occlusive disease.

\section{Preprocedural Evaluation}

Initial evaluation of the patient begins with a focused history and physical examination, and review of any available imaging. Cross-sectional imaging with either contrast-enhanced computed tomography (CT) or magnetic resonance (MR) is extremely helpful to delineate the anatomy of the lesion and assist in procedural planning. Both CT and MR can be used to diagnose any underlying pathology, including tumor size and localization. ${ }^{1}$ Thin section, contrast-enhanced CT is especially useful to determine the amount of venous stenosis/occlusion, identify prominent collateral vessels, and identify anatomic landmarks to avoid during recanalization. Routine laboratory tests including blood counts and serum chemistries are obtained on the day of the procedure. Optimal laboratory values include a serum creatinine $<1.5 \mathrm{mg} / \mathrm{dL}$ with an estimated glomerular filtration rate $>60$, and a platelet count of $>50,000 / \mathrm{dL}$. In the outpatient setting, the authors recommend holding or reversing anticoagulation for this procedure so the interventionalist can administer intraprocedural unfractionated heparin and monitor anticoagulation levels during the procedure.

\section{Procedure}

When procedures are expected to be prolonged or challenging, the authors typically perform them with general anesthesia for patient comfort, however, moderate sedation using intravenous fentanyl and midazolam can also be used. The patient is positioned supine on the fluoroscopic table and depending on preference for vascular access, the upper extremity, and neck ipsilateral to the venous occlusion is prepped using the standard sterile technique. The right groin is also prepped as access into the right common femoral vein (CFV) is routinely gained during the procedure to assist in recanalization. Perioperative intravenous antibiotics are administered ( $1 \mathrm{~g}$ cefazolin), and systemic anticoagulation with unfractionated heparin is routine.
Issue Theme Morbidity and Mortality in Interventional Radiology: Case Series; Guest Editors, Brian Funaki, MD, FSIR, and Charles E. Ray, Jr., MD, PhD, FSIR
Copyright @ 2015 by Thieme Medical Publishers, Inc., 333 Seventh Avenue, New York, NY 10001, USA. Tel: +1(212) 584-4662.
DOI http://dx.doi.org/ 10.1055/s-0034-1396966. ISSN 0739-9529. 
Depending on the location of the occlusion, under direct ultrasound guidance venous access into the ipsilateral upper extremity or neck is achieved with a 21 gauge micropuncture set and a $30-\mathrm{cm} 6 \mathrm{~F}$ vascular sheath (Cook Inc., Bloomington, IN) is placed. The long sheath not only provides an adequate sized working conduit, but also provides stability and stiffness for the system. Venography of the involved vessels adjacent to the lesion is performed to delineate the anatomy of the occlusion for procedural planning. Simultaneous venography performed through sheaths or catheters on each side of the occlusion is also useful to evaluate the length of the occlusion. Through the $6 \mathrm{~F}$ vascular sheath and over an angled Glidewire (Terumo Medical Corp., Somerset, NJ), an angled 5F Kumpe catheter (Cook Inc.) is advanced to the stump of the venous occlusion. Under fluoroscopic guidance, the Glidewire is advanced out the tip of the catheter toward the occlusion, using graded force. Gently probing with the Glidewire may be adequate to cross severe stenoses or acute occlusions. If the wire fails to traverse the occlusion, incrementally greater force is used with the wire. Advancing the angled catheter and sheath against the occlusion will give the Glidewire more stiffness and will direct the tip of the wire directly into the lesion. If the tip of the wire will not advance into the lesion after these techniques the lesion will be probed with the stiffer back end of the Glidewire. The advantage is that as the wire exits the catheter tip, it creates a very sharp point; however, it is very easy to place the wire in the extravascular space with this technique and caution should be used. The authors will frequently use a combination of the tip and the "back end" of the Glidewire by alternating between the two for longer recanalizations; this is performed by pushing into the occlusion with the "back end" of the Glidewire and, as incremental distance through the occlusion is gained, advance the catheter is further advanced into the occlusion, and the wire is again reversed to probe with the tip of the Glidewire. A loop snare (eV3 Endovascular Inc., Plymouth, MN) or pigtail catheter can be placed at the distal (downstream) margin of the occlusion via the CFV vascular access to provide a visible target for wire placement. Frequent rotation of the fluoroscopic unit is required to ensure that recanalization is progressing in the correct direction.

Once the lesion is crossed, the $5 \mathrm{~F}$ catheter is advanced past the occlusion over the wire, and intraluminal position is verified with aspiration of blood. Once the blood is aspirated,
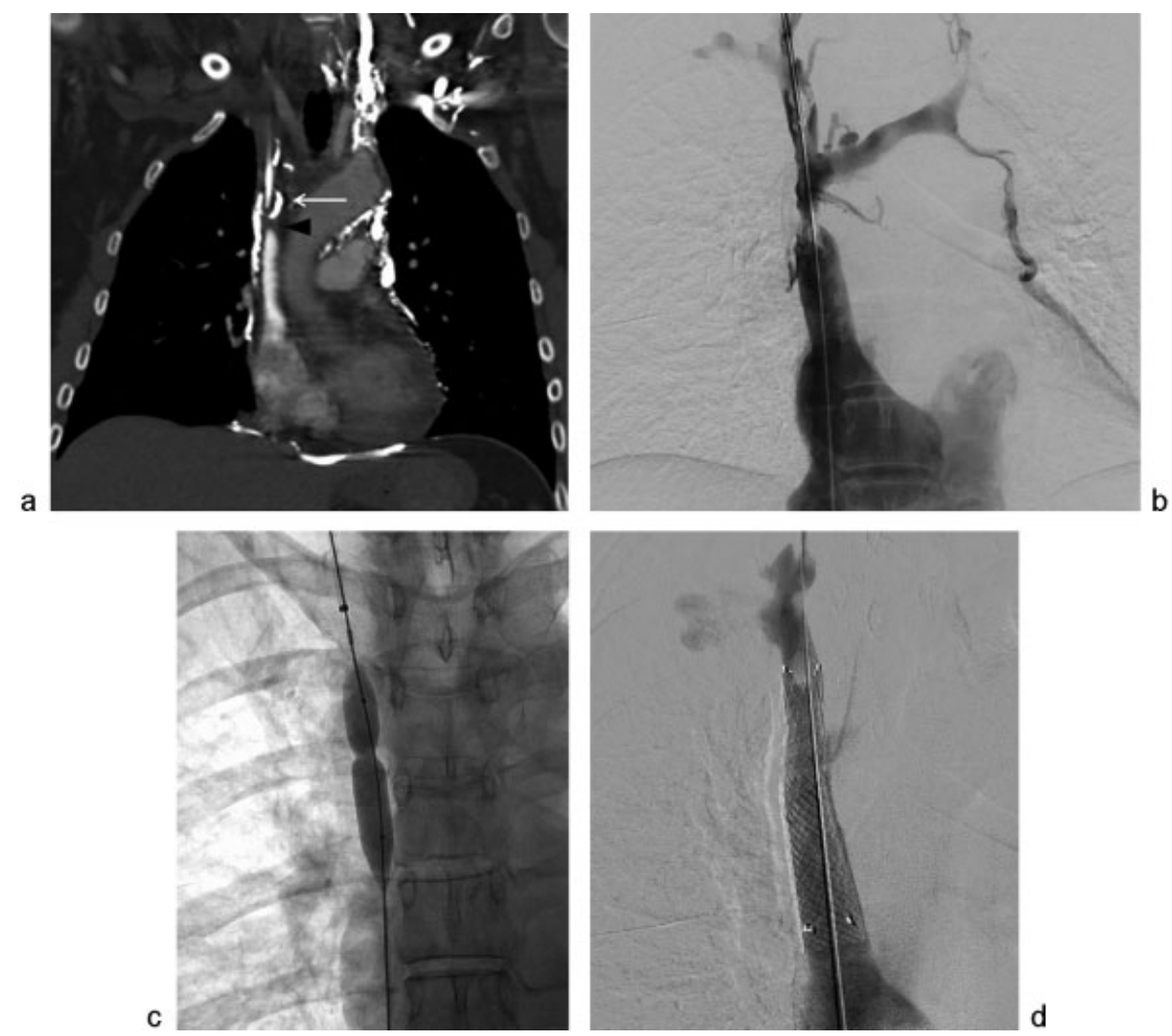

Fig. 1 A 55-year-old woman with a history of ovarian cancer and indwelling port-a-cath device who presented with progressive onset of facial swelling, plethora, and hoarseness. (a) Coronal reformatted contrast-enhanced CT of the thorax demonstrates numerous neck and mediastinal collaterals with thrombus surrounding the distal tip of the port-a-cath (arrow) and severe SVC stenosis (black arrowhead). (b) SVC venogram demonstrates focal severe stenosis with filling of collaterals; the lesion had been successfully traversed easily with a catheter and Glidewire (Terumo Medical Corp., Somerset, NJ). (c) During venoplasty, inflation of a 10-mm balloon demonstrates a focal waist with initial venoplasty. (d) Post-stent placement SVC venogram demonstrates in-line flow into the right atrium; no collaterals are seen, suggested a good hemodynamic response. CT, computed tomography; SVC, superior vena cava. 
a gentle venogram is performed to confirm reentry into the vessel lumen. As it is not uncommon for the catheter to not follow the wire through parts of the tract, the use of an exchange length wire is critical to allow snaring the wire from the CFV access and pulling the wire for a through-andthrough access; through-and-through access allows a stiff rail that almost always allows for advancing a catheter through an occlusion. If a conventional catheter still does not pass, a tapered crossing catheter such as a Quick Cross (Spectranetics, Colorado Springs, CO) may be helpful. When the catheter itself is externalized at the groin access site, an exchange length stiff wire (260 cm Amplatz; Cook Inc.) can be positioned across the lesion. Venoplasty before the stent placement is typically performed; the authors usually underdilate the stenosis with a 4 - to $5-\mathrm{mm}$ balloon; this is performed to allow the passage of a stent. Aggressive overdilation is avoided initially, particularly when recanalizing the SVC to avoid a potentially fatal rupture into the pericardial space with resultant pericardial tamponade. When making measurements for stent placement, a $5 \mathrm{~F}$ marker pigtail catheter (Cook Inc.) is placed over the wire, and a simultaneous venogram is performed through the sheaths above and below the occlusion. Once measured, a selfexpanding nitinol stent is deployed across the lesion, and venoplasty is performed with increasing sizes of balloons and intermittent venography to evaluate for residual stenosis/thrombosis, reduction of collateral blood flow, and to assess for complications (-Fig. 1). If contrast extravasation is detected into the pericardial space, anticoagulation is reversed, balloon tamponade across the prior obstruction is performed, and ultrasound is made available for potential emergent pericardiocentesis.

In the authors practice, initial attempts at chronic venous recanalizations are made with a catheter and hydrophilic wire, as many occlusions can be traversed with this method. If the lesion is recalcitrant to hydrophilic wire traversal, an attempt at sharp recanalization (-Fig. 2) using a 15- to 65-cm 21-gague Chiba tipped needle (Cook Inc.) is made. When positioning the needle toward the occlusion, care should be taken not to pierce the sheath with the Chiba tip. The needle is placed in coaxial fashion through the vascular dilator included with the sheath, and the needle tip is exposed only once the dilator reaches the lesion. Short, purposeful, and quick passes with the needle are made. A marker pigtail catheter or snare placed at the opposite side of the occlusion is mandatory when attempting sharp recanalization, and frequent image intensifier rotation will help to evaluate the needle tip position relative to the target. Aspiration is performed while withdrawing the needle and, if blood is aspirated, contrast is gently injected to evaluate for intravascular positioning. A slight bend or graduated curve to the distal needle can be formed for additional direction control. If sharp recanalization is unsuccessful or thought to be unsafe for the patient, a 250$\mathrm{cm} \mathrm{0.035-in.} \mathrm{PowerWire} \mathrm{Radiofrequency} \mathrm{(RF)} \mathrm{Guidewire}$ (Baylis Medical, Montreal, Québec, Canada) can be used for more challenging or long segment recanalizations (-Fig.3). Through the indwelling venous guide sheath, the RF wire is
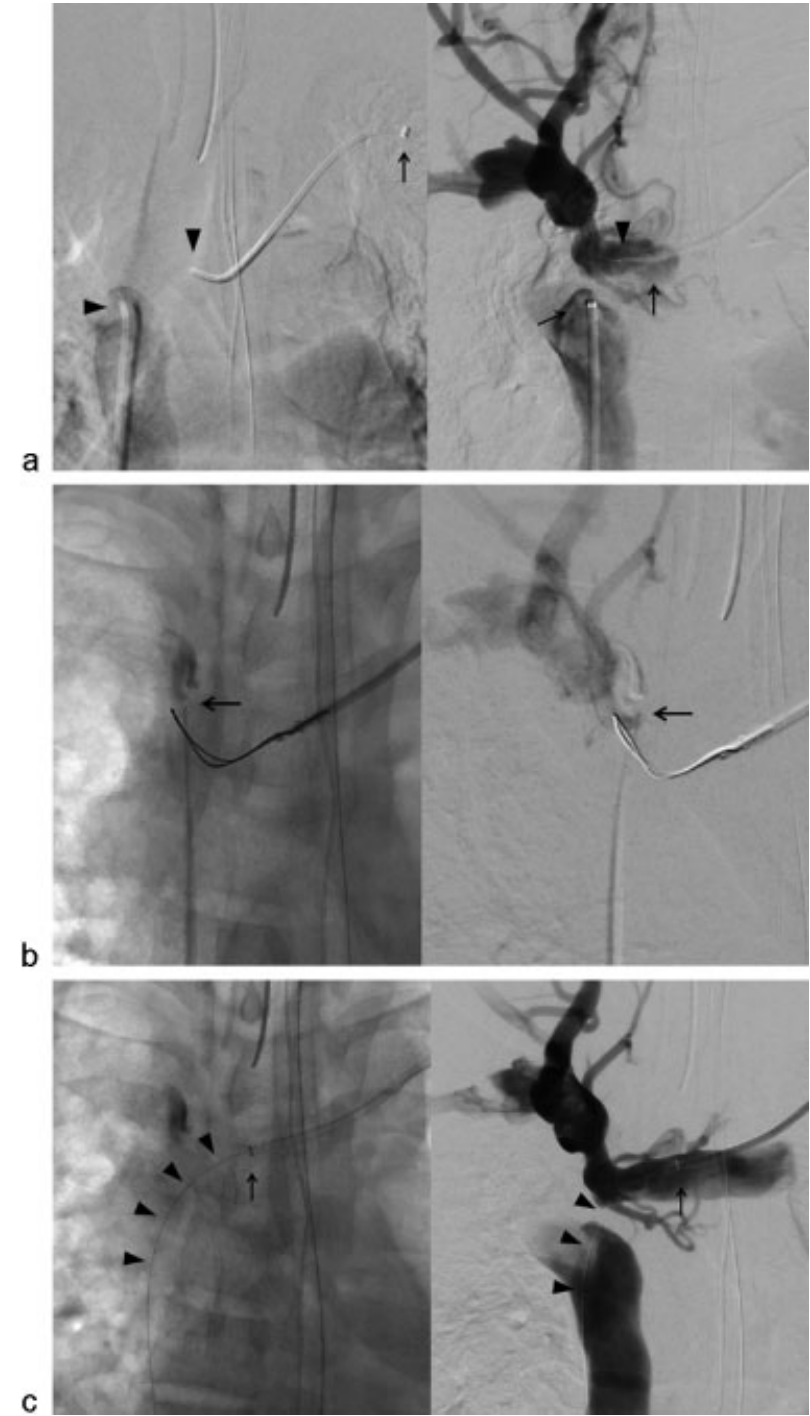

Fig. 2 A 58-year-old woman with end-stage renal disease on chronic hemodialysis presents with a 2 month history of left upper extremity and facial swelling. (a) Tandem left-sided brachiocephalic and SVC venograms performed through angled catheters (arrowheads) demonstrate short-segment occlusion of the SVC with numerous neck and mediastinal collaterals (sheath tips-arrows). (b) Fluoroscopic spot image and venogram after sharp recanalization of the occlusion from a caudal approach with a 65-cm 21-gauge Chiba-tipped needle (Cook Inc., Bloomington, IN) demonstrates intravascular position of the needle tip (arrows). (c) Fluoroscopic spot image and tandem venogram shows "through-and-through" access after snaring a microwire (arrowheads) through the sheath (arrow); this was exchanged for an exchange length 0.035 -in. wire to perform balloon venoplasty and stent placement. SVC, superior vena cava.

advanced through the angled catheter toward a target snare in short (3-5 mm) increments. A 25 Watt power is applied for 2 second pulses with a dedicated RF generator, under fluoroscopic guidance. Once the wire is advanced, through the occlusion the tip of the $250 \mathrm{~cm}$ RF wire can be snared or the $5 \mathrm{~F}$ catheter can be advanced over the wire. Once "through-and-through" access is achieved, balloon venoplasty and stent placement is similar to what is previously described. 


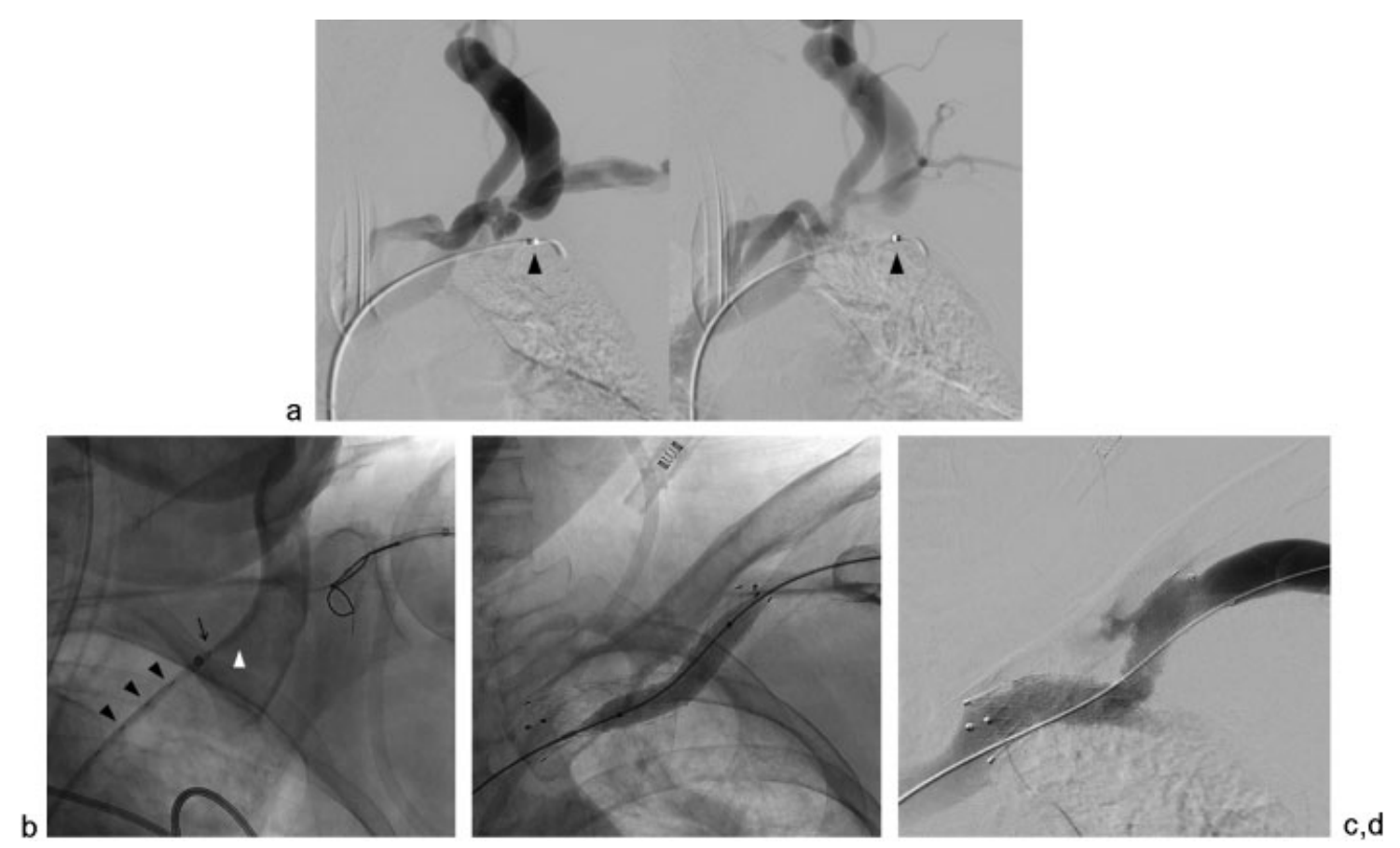

Fig. 3 A 60-year-old woman with end-stage renal disease on chronic hemodialysis who presents with progressive left-sided neck and facial swelling. (a) Early and late left-sided cephalic arch venograms demonstrate short segment central occlusion with reconstitution of the brachiocephalic vein by a large tortuous neck collateral (sheath/angled catheter placed in the ipsilateral subclavian vein-arrowhead). (b) Fluoroscopic spot image demonstrates RF wire (arrowheads) and RF wire tip (arrow) positioned in an angled catheter (white arrowhead); loop snare positioned in the patent portion of the cephalic arch used as a target. (c) Fluoroscopic spot image shows 8-mm balloon catheter venoplasty after "through-and-through" access gained and 14-mm stent (LifeStar-Bard Medical, Covington, GA) placement. (d) Postprocedure cephalic arch venogram demonstrates improved in-line flow through stented segment with reduction of collateral filling. RF, radiofrequency.

\section{Postprocedural Evaluation}

Postprocedurally, the patients are transferred to the postanesthesia care unit for cardiovascular monitoring. It is typical for patients to be on bed rest precautions for 2 to 4 hours due to the CFV puncture and the systemic intraprocedural anticoagulation. If the CFV was not accessed, the bed rest precaution time is reduced. If flow was restored after the procedure, systemic anticoagulation is not typically necessary unless there is residual thrombus. Low-dose aspirin ( $81 \mathrm{mg}$ ) is given for several months to allow the coverage of the stent with neoendothelium. In the authors practice, patients are seen back in a clinic setting 1 to 2 weeks after the procedure.

\section{Summary}

Chronic central venous occlusion can be a debilitating condition for patients. Malignancy, such as NSCLC, is the most common etiology although indwelling intravascular devices, prior thoracic radiation therapy, and fibrosing mediastinitis can cause chronic central venous occlusions as well. Treat- ment goals are focused on symptomatic relief and, if the etiology is malignancy, treatment is often palliative. Percutaneous recanalization and endovascular stenting of chronic occlusions is a minimally invasive technique that can be performed in an outpatient setting, often resulting in immediate relief of symptoms.

\section{References}

1 Lepper PM, Ott SR, Hoppe H, et al. Superior vena cava syndrome in thoracic malignancies. Respir Care 2011;56(5):653-666

2 Mauro MA, Murphy K, Thomson KR, Venbrux AC, Zollikofer CL. Image-Guided Interventions. Philadelphia, PA: Saunders Elsevier; 2008:1055

3 Parish JM, Marschke RF Jr, Dines DE, Lee RE. Etiologic considerations in superior vena cava syndrome. Mayo Clin Proc 1981; 56(7):407-413

4 Rice TW, Rodriguez RM, Light RW. The superior vena cava syndrome: clinical characteristics and evolving etiology. Medicine (Baltimore) 2006;85(1):37-42

5 Wudel LJ Jr, Nesbitt JC. Superior vena cava syndrome. Curr Treat Options Oncol 2001;2(1):77-91 